\title{
Existence Results for New Weak and Strong Mixed Vector Equilibrium Problems on Noncompact Domain
}

\author{
Ali Farajzadeh, ${ }^{1}$ Kasamsuk Ungchittrakool, ${ }^{2,3,4}$ and Apisit Jarernsuk ${ }^{2}$ \\ ${ }^{1}$ Department of Mathematics, Razi University, Kermanshah 67149, Iran \\ ${ }^{2}$ Department of Mathematics, Faculty of Science, Naresuan University, Phitsanulok 65000, Thailand \\ ${ }^{3}$ Centre of Excellence in Mathematics, CHE, Si Ayutthaya Road, Bangkok 10400, Thailand \\ ${ }^{4}$ Research Center for Academic Excellence in Nonlinear Analysis and Optimizations, Faculty of Science, Naresuan University,
} Phitsanulok 65000, Thailand

Correspondence should be addressed to Kasamsuk Ungchittrakool; kasamsuku@nu.ac.th

Received 22 March 2015; Accepted 10 June 2015

Academic Editor: Tomonari Suzuki

Copyright (C) 2015 Ali Farajzadeh et al. This is an open access article distributed under the Creative Commons Attribution License, which permits unrestricted use, distribution, and reproduction in any medium, provided the original work is properly cited.

We introduce and consider two new mixed vector equilibrium problems, that is, a new weak mixed vector equilibrium problem and a new strong mixed vector equilibrium problem which are combinations of certain vector equilibrium problems, and vector variational inequality problems. We prove existence results for the problems in noncompact setting.

\section{Introduction}

There are several problems of applied and substantial interest in optimization, economics, and engineering that are related to equilibrium in their nature. The equilibrium problem was introduced and studied by [1] as a generalization of variational inequality problem. It has been shown that the equilibrium problem provides a natural, novel, and unified framework to study a wide class of problems arising in nonlinear analysis, optimization, economics, finance, and game theory. The equilibrium problem includes many mathematical problems as particular cases such as mathematical programming problems, complementarity problems, variational inequality problems, fixed point problems, minimax inequality problems, and Nash equilibrium problems in noncooperative games; see [1-4].

Let $X$ be a Hausdorff topological vector space, let $K$ be a subset of $X$, and let $f: K \times K \rightarrow \mathbb{R}$ be a mapping with $f(x, x)=0$. The classical, scalar-valued equilibrium problem deals with the existence of $\tilde{x} \in K$ such that

$$
f(\widetilde{x}, y) \geq 0 ; \quad \forall y \in K .
$$

Moreover, in the case of vector valued mappings, let $Y$ be another Hausdorff topological vector space, $C \subset Y$, a convex cone with nonempty interior. Given a vector mapping $f$ : $K \times K \rightarrow Y$, then the problem of finding $\tilde{x} \in K$ such that

$$
f(\widetilde{x}, y) \notin-\operatorname{int} C ; \quad \forall y \in K
$$

is called weak equilibrium problem and the point $\tilde{x} \in K$ is called weak equilibrium point, where $\operatorname{int} C$ denotes the interior of the cone $C$ in $Y$. In 2014, Rahaman and Ahmad [5] considered two types of mixed vector equilibrium problems which were combinations of a vector equilibrium problem and a vector variational inequality problem. Remark that $C \mathrm{C}$ $Y$ is a pointed closed convex cone with nonempty interior; that is, $\operatorname{int} C \neq \emptyset$. The partial ordering induced by $C$ on $Y$ is denoted by $\leq_{C}$ and is defined by $x \leq_{C} y$ if and only if $y-x \in C$. Let $f: K \times K \rightarrow Y$ and $T: X \rightarrow L(X, Y)$ be two mappings, where $L(X, Y)$ is the space of all linear continuous mappings from $X$ to $Y$. Here $\langle T(x), y\rangle$ denotes the evaluation of the linear mapping $T(x)$ at $y$. They considered the following two problems.

Find $\tilde{x} \in K$ such that

$$
\begin{aligned}
& f(\tilde{x}, y)+\langle T(\widetilde{x}), y-\tilde{x}\rangle \notin-\operatorname{int} C ; \quad \forall y \in K, \\
& f(\tilde{x}, y)+\langle T(\widetilde{x}), y-\tilde{x}\rangle \notin-C \backslash\{0\} ; \quad \forall y \in K .
\end{aligned}
$$


It is clear that the solution set of (4) is a subset of the solution set of (3). Also if we consider $Y=\mathbb{R}^{2}$ and $C=$ $\{(x, x): x \in \mathbb{R}\}$, then int $C=\emptyset$ and the solution set of $(3)$ is always the whole set $K$. They called problem (3) weak mixed vector equilibrium problem and problem (4) strong mixed vector equilibrium problem. Problems (3) and (4) are unified models of several known problems used in applied sciences, for instance, vector variational inequality problem, vector complementarity problem, vector optimization problem, and vector saddle point problem; see, for example, $[3,6-10]$ and references therein.

With the inspiration from the notice of some characteristics of the mappings of the original problem, we are interested and motivated in the development of the existing problems to the new weak mixed vector equilibrium problem and the new strong mixed vector equilibrium as follows.

Find $\tilde{x} \in K$ such that

$$
\begin{aligned}
& f(\tilde{x}, y)+\langle T(\tilde{x}), y-\tilde{x}\rangle+\tau(\tilde{x}, y) \\
& \quad+\langle y-\tilde{x}, D \tilde{x}-D z\rangle \notin-\operatorname{int} C ; \quad \forall y \in K, \\
& f(\tilde{x}, y)+\langle T(\tilde{x}), y-\tilde{x}\rangle+\tau(\tilde{x}, y) \\
& \quad+\langle y-\tilde{x}, D \tilde{x}-D z\rangle \notin-C \backslash\{0\} ; \quad \forall y \in K,
\end{aligned}
$$

where $\tau: K \times K \rightarrow Y$ is a bifunction, $D: X \rightarrow$ $L(X, Y)$, and $z \in X$. For a more comprehensive bibliography on vector equilibrium problems, vector variational inequality problems, and their generalizations, we refer to volume edited by [3]. Our results generalize the results obtained by [1] and therefore the results of Fan [11] for vector valued mappings. For more details, we refer to $[6,12,13]$. As the underlying set $K$ is noncompact, therefore we use only a very weak coercivity condition, that is, coercing family.

\section{Preliminaries}

The following definitions and results are needed in the sequel. Let $X$ and $Y$ be two Hausdorff topological vector spaces, $K$ a subset of $X$, and $C$ a pointed convex cone of $Y$.

Definition 1. Let $g: K \rightarrow Y$ be a mapping. Then $g$ is said to be $C$-convex, if for all $x, y \in K$ and $\lambda \in[0,1]$

$$
g(\lambda x+(1-\lambda) y) \leq_{C} \lambda g(x)+(1-\lambda) g(y),
$$

which implies that

$$
g(\lambda x+(1-\lambda) y) \in \lambda g(x)+(1-\lambda) g(y)-C .
$$

Definition 2. Let $g: K \rightarrow Y$ be a mapping.

(i) $g$ is said to be lower semicontinuous with respect to $C$ at a point $x_{0} \in K$, if for any neighborhood $V$ of $g\left(x_{0}\right)$ in $Y$ there exists a neighborhood $U$ of $x_{0} \in X$ such that $g(U \cap K) \subseteq V+C$;

(ii) $g$ is said to be upper semicontinuous with respect to $C$ at a point $x_{0} \in K$, if $g(U \cap K) \subseteq V-C$;

(iii) $g$ is said to be continuous with respect to $C$ at a point $x_{0} \in K$, if it is lower semicontinuous and upper semicontinuous with respect to $C$ at that point.
Remark 3. If $g$ is lower semicontinuous (upper semicontinuous or continuous, resp.) with respect to $C$ at any point of $K$, then $g$ is lower semicontinuous (upper semicontinuous or continuous, resp.) with respect to $C$ on $K$.

Definition 4. A mapping $f: K \times K \rightarrow Y$ is said to be $C$ monotone, if for all $x, y \in K$

$$
f(x, y)+f(y, x) \in-C .
$$

Lemma 5 (see [10]). If $g$ is a lower semicontinuous mapping with respect to $C$, then the set

$$
\{x \in K: g(x) \notin \operatorname{int} C\}
$$

is closed in $\mathrm{K}$.

Lemma 6 (see [14]). Let $(Y, C)$ be an ordered topological vector space with a pointed closed convex cone $C$. Then for all $x, y \in Y$ we have that

(i) $y-x \in \operatorname{int} C$ and $y \notin \operatorname{int} C$ imply $x \notin \operatorname{int} C$;

(ii) $y-x \in C$ and $y \notin \operatorname{int} C$ imply $x \notin \operatorname{int} C$;

(iii) $y-x \in-\operatorname{int} C$ and $y \notin-\operatorname{int} C$ imply $x \notin-\operatorname{int} C$;

(iv) $y-x \in-C$ and $y \notin-\operatorname{int} C$ imply $x \notin-\operatorname{int} C$.

Definition 7 (see [15]). Consider a subset $K$ of a topological vector space and a topological space $Y$. A family $\left\{\left(C_{i}, Z_{i}\right)\right\}_{i \in I}$ of pair of sets is said to be coercing for a multivalued mapping $F: K \rightarrow 2^{Y}$ if and only if

(i) for each $i \in I, C_{i}$ is contained in a compact convex subset of $K$ and $Z_{i}$ is a compact subset of $Y$;

(ii) for each $i, j \in I$ there exists $k \in I$ such that $C_{i} \cup C_{j} \subseteq$ $C_{k}$;

(iii) for each $i \in I$ there exists $k \in I$ with $\bigcap_{x \in C_{k}} F(x) \subseteq Z_{i}$.

Definition 8. Let $K$ be a nonempty convex subset of a topological vector space $X$. A multivalued mapping $G: K \rightarrow$ $2^{X}$ is said to be KKM mapping, if, for every finite subset $\left\{x_{i}\right\}_{i \in I}$ of $K$,

$$
\operatorname{Co}\left\{x_{i}: i \in I\right\} \subseteq \bigcup_{i \in I} F\left(x_{i}\right)
$$

where $\operatorname{Co}\left\{x_{i}: i \in I\right\}$ denotes the convex hull of $\left\{x_{i}\right\}_{i \in I}$.

Theorem 9 (see [15]). Let $X$ be a Hausdorff topological vector space, $Y$ a convex subset of $X, K$ a nonempty subset of $Y$, and $F: K \rightarrow 2^{Y}$ a KKM mapping with compactly closed values in $Y$ (i.e., for all $x \in K, F(x) \cap Z$ is closed for every compact set $Z$ of $Y)$. If $F$ admits a coercing family, then

$$
\bigcap_{x \in K} F(x) \neq \emptyset
$$

Condition $(C)$ : we say that the cone $C$ satisfies Condition $(C)$, if there is a pointed convex closed cone $\widetilde{C}$ such that $C \backslash\{0\} \subseteq$ int $\widetilde{C}$. 


\section{Main Results}

In this section, we prove the following existence results for new weak and strong mixed vector equilibrium problems (5) and (6) for noncompact domain.

Theorem 10. Let $K$ be a nonempty closed convex subset of a Hausdorff topological vector space $X, Y$ a Hausdorff topological vector space, and $C$ a closed convex pointed cone in $Y$ with $\operatorname{int} C \neq \emptyset$. Let $f: K \times K \rightarrow Y, \tau: K \times K \rightarrow Y$, $T: K \rightarrow L(X, Y)$, and $D: X \rightarrow L(X, Y)$ be four mappings satisfying the following conditions:

(i) $f$ and $\tau$ are $C$-monotone.

(ii) $f(x, x)=0$, and $\tau(x, x)=0$ for all $x \in K$.

(iii) For any fixed $x, y \in K, t \in[0,1] \mapsto f(t y+(1-t) x, y) \in$ $Y$ and $t \in[0,1] \mapsto \tau(t y+(1-t) x, y) \in Y$ are upper semicontinuous with respect to $C$ at $t=0$.

(iv) For any fixed $x \in K, f(x, \cdot), \tau(x, \cdot): K \rightarrow Y$ are $C$ convex, lower semicontinuous with respect to $C$ on $K$.

(v) $D$ and $T$ are upper semicontinuous with respect to $C$ with nonempty closed values.

(vi) There exists a family $\left\{C_{i}, Z_{i}\right\}_{i \in I}$ satisfying conditions ( $i$ ) and (ii) of Definition 7 and the following condition: For each $i \in I$, there exists $k \in I$ such that

$$
\begin{aligned}
& \{x \in K: f(y, x)-\langle T x, y-x\rangle+\tau(y, x) \\
& \left.\quad-\langle y-x, D x-D z\rangle \notin \operatorname{int} C, \forall y \in C_{k}\right\} \subset Z_{i} .
\end{aligned}
$$

Then, there exists a point $\tilde{x} \in K$ such that

$$
\begin{aligned}
& f(\tilde{x}, y)+\langle T(\tilde{x}), y-\tilde{x}\rangle+\tau(\tilde{x}, y) \\
&+\langle y-x, D x-D z\rangle \notin-\operatorname{int} C ; \quad \forall y \in K .
\end{aligned}
$$

For the proof of Theorem 10, we need the following proposition, for which the assumptions remain the same as in Theorem 10.

Proposition 11. The following two problems are equivalent:

(i) Find $\tilde{x} \in K$ such that $f(y, \tilde{x})-\langle T(\tilde{x}), y-\tilde{x}\rangle+\tau(y, \tilde{x})-$ $\langle y-\tilde{x}, D \tilde{x}-D z\rangle \notin \operatorname{int} C ; \forall y \in K$.

(ii) Find $\tilde{x} \in K$ such that $f(\tilde{x}, y)+\langle T(\tilde{x}), y-\tilde{x}\rangle+\tau(\tilde{x}, y)+$ $\langle y-\tilde{x}, D \tilde{x}-D z\rangle \notin-\operatorname{int} C ; \forall y \in K$.

Proof. Suppose (i) holds. Then, for fixed $y \in K$, set $x_{t}=t y+$ $(1-t) \widetilde{x}$, for $t \in[0,1]$. It is clear that $x_{t} \in K$ for all $t \in[0,1]$ and hence

$$
\begin{gathered}
f\left(x_{t}, \tilde{x}\right)-\left\langle T(\tilde{x}), x_{t}-\tilde{x}\right\rangle+\tau\left(x_{t}, \tilde{x}\right) \\
-\left\langle x_{t}-\tilde{x}, D \tilde{x}-D z\right\rangle \notin \operatorname{int} C .
\end{gathered}
$$

Since $f(x, x)=0$ and $f(x, \cdot)$ is $C$-convex, we have

$$
\begin{aligned}
0 & =f\left(x_{t}, x_{t}\right) \leq_{C} t f\left(x_{t}, y\right)+(1-t) f\left(x_{t}, \tilde{x}\right) \\
& \Longrightarrow t f\left(x_{t}, y\right)+(1-t) f\left(x_{t}, \tilde{x}\right) \in C .
\end{aligned}
$$

On the other hand, the convexity of $\tau$ in the second variable implies that

$$
\begin{aligned}
0 & =\tau\left(x_{t}, x_{t}\right) \leq_{C} t \tau\left(x_{t}, y\right)+(1-t) \tau\left(x_{t}, \tilde{x}\right) \\
& \Longrightarrow t \tau\left(x_{t}, y\right)+(1-t) \tau\left(x_{t}, \tilde{x}\right) \in C .
\end{aligned}
$$

Also,

$$
\begin{aligned}
\left\langle T(\tilde{x}), x_{t}-\tilde{x}\right\rangle= & t\langle T(\tilde{x}), y-\tilde{x}\rangle \\
\Longrightarrow & (1-t) t\langle T(\tilde{x}), y-\tilde{x}\rangle \\
& \quad-(1-t)\left\langle T(\tilde{x}), x_{t}-\tilde{x}\right\rangle=0, \\
\left\langle x_{t}-\tilde{x}, D \tilde{x}-D z\right\rangle= & t\langle y-\tilde{x}, D \tilde{x}-D z\rangle \\
\Longrightarrow & (1-t) t\langle y-\tilde{x}, D \tilde{x}-D z\rangle \\
& \quad-(1-t)\left\langle x_{t}-\tilde{x}, D \tilde{x}-D z\right\rangle \\
= & 0 .
\end{aligned}
$$

Combining (16), (17), and (18) we obtain

$$
\begin{aligned}
t & \left(f\left(x_{t}, y\right)+\tau\left(x_{t}, y\right)\right)+(1-t)\left\{f\left(x_{t}, \tilde{x}\right)+\tau\left(x_{t}, \tilde{x}\right)\right. \\
& \left.-\left\langle T(\widetilde{x}), x_{t}-\tilde{x}\right\rangle-\left\langle x_{t}-\widetilde{x}, D \widetilde{x}-D z\right\rangle\right\}+(1-t) \\
& +t\{\langle T(\widetilde{x}), y-\tilde{x}\rangle \\
& +\langle y-\widetilde{x}, D \tilde{x}-D z\rangle\} \in C,
\end{aligned}
$$

for all $t \in[0,1]$. It is not hard to see that (19) is equivalent to

$$
\begin{aligned}
& (1-t)\left\{f\left(x_{t}, \tilde{x}\right)+\tau\left(x_{t}, \tilde{x}\right)-\left\langle T(\widetilde{x}), x_{t}-\tilde{x}\right\rangle\right. \\
& \left.\quad-\left\langle x_{t}-\tilde{x}, D \tilde{x}-D z\right\rangle\right\}-\left(-t\left(f\left(x_{t}, y\right)+\tau\left(x_{t}, y\right)\right)\right. \\
& -(1-t) t\{\langle T(\widetilde{x}), y-\tilde{x}\rangle+\langle y-\widetilde{x}, D \tilde{x}-D z\rangle\})
\end{aligned}
$$

$\in C$.

By using (15) and (20) and (ii) of Lemma 6, we have

$$
\begin{aligned}
t & \left(f\left(x_{t}, y\right)+\tau\left(x_{t}, y\right)\right) \\
& +(1-t) t\{\langle T(\widetilde{x}), y-\tilde{x}\rangle+\langle y-\widetilde{x}, D \widetilde{x}-D z\rangle\} \\
& \notin-\operatorname{int} C \Longrightarrow f\left(x_{t}, y\right)+\tau\left(x_{t}, y\right) \\
& +(1-t)\langle T(\widetilde{x}), y-\tilde{x}\rangle \\
& +(1-t)\langle y-\tilde{x}, D \tilde{x}-D z\rangle \notin-\operatorname{int} C,
\end{aligned}
$$

$\forall t \in(0,1]$.

By condition (iii) of Theorem 10 as $t \mapsto f(t y+(1-t) x, y)$ and $t \mapsto \tau(t y+(1-t) x, y)$ are upper semicontinuous with respect to $C$ at $t=0$, therefore from (21) we have

$$
\begin{array}{r}
f(\tilde{x}, y)+\langle T(\tilde{x}), y-\tilde{x}\rangle+\tau(\tilde{x}, y) \\
+\langle y-\tilde{x}, D \tilde{x}-D z\rangle \notin-\operatorname{int} C,
\end{array}
$$

and hence (ii) holds. 
Conversely, we assume that (ii) holds. In order to prove (i), on the contrary suppose that there exists a point $\tilde{y} \in K$ such that

$$
\begin{aligned}
f & (\tilde{y}, \tilde{x})-\langle T(\tilde{x}), \tilde{y}-\tilde{x}\rangle+\tau(\tilde{y}, \tilde{x}) \\
& -\langle\tilde{y}-\tilde{x}, D \tilde{x}-D z\rangle \in \operatorname{int} C \Longrightarrow f(\tilde{y}, \tilde{x}) \\
& +\tau(\tilde{y}, \tilde{x})-\langle\widetilde{y}-\tilde{x}, D \tilde{x}-D z\rangle=\langle T(\widetilde{x}), \tilde{y}-\tilde{x}\rangle \\
& +w,
\end{aligned}
$$

for some $w \in \operatorname{int} C$. have

On the other hand, since $f$ and $\tau$ are $C$-monotone, we

$$
\begin{aligned}
f(\tilde{x}, \tilde{y})+f(\tilde{y}, \tilde{x}) & \epsilon-C \Longrightarrow f(\tilde{y}, \tilde{x}) \\
& =-f(\widetilde{x}, \tilde{y})-v,
\end{aligned}
$$

for some $v \in C$ and

$$
\tau(\tilde{x}, \tilde{y})+\tau(\tilde{y}, \tilde{x}) \in-C \Longrightarrow \tau(\tilde{y}, \tilde{x})=-\tau(\tilde{x}, \tilde{y})-u,
$$

for some $u \in C$. Combining (23), (24), and (25), we have

$$
\begin{aligned}
f & (\tilde{x}, \tilde{y})+\langle T(\tilde{x}), \tilde{y}-\tilde{x}\rangle+\tau(\tilde{x}, \tilde{y}) \\
& +\langle\tilde{y}-\tilde{x}, D \tilde{x}-D z\rangle=-w-v-u \in-\operatorname{int} C,
\end{aligned}
$$

which contradicts assumption (ii). Therefore (i) holds.

Now, we are able to prove Theorem 10 which has the following details.

Proof. For each $y \in K$, consider the set

$$
\begin{aligned}
& F(y)=\{x \in K: f(y, x)-\langle T(x), y-x\rangle+\tau(y, x) \\
& \quad-\langle y-x, D x-D z\rangle \notin \operatorname{int} C\} .
\end{aligned}
$$

By Lemma 5, $F(y)$ is closed in $K$ and hence $F$ has compactly closed values in $K$.

Now, we show that $F$ is a KKM map. For this, let $\left\{y_{i}: i \in I\right\}$ be a finite subset of $K$ and $u \in \operatorname{Co}\left\{y_{i}: i \in I\right\}$.

We claim that

$$
\operatorname{Co}\left\{y_{i}: i \in I\right\} \subseteq \bigcup_{i \in I} F\left(y_{i}\right) .
$$

On the contrary, suppose that $u \notin \bigcup_{i \in I} F\left(y_{i}\right)$. As $u \in \operatorname{Co}\left\{y_{i}\right.$ : $i \in I\}$, we have $u=\sum_{i \in I} \lambda_{i} y_{i}$ with $\lambda_{i} \geq 0$ and $\sum_{i \in I} \lambda_{i}=1$. This follows that

$$
\begin{gathered}
f\left(y_{i}, u\right)-\left\langle T(u), y_{i}-u\right\rangle+\tau\left(y_{i}, u\right) \\
-\left\langle y_{i}-x, D x-D z\right\rangle \in \operatorname{int} C .
\end{gathered}
$$

Since int $C$ is convex, therefore

$$
\begin{aligned}
\sum_{i \in I} \lambda_{i}\left\{f\left(y_{i}, u\right)-\left\langle T(u), y_{i}-u\right\rangle+\tau\left(y_{i}, u\right)\right. \\
\left.\quad-\left\langle y_{i}-x, D x-D z\right\rangle\right\} \in \operatorname{int} C .
\end{aligned}
$$

Since $f(x, \cdot)$ is $C$-convex and $C$-monotone, we have

$$
\begin{aligned}
\sum_{i \in I} \lambda_{i} f\left(y_{i}, u\right) & \leq_{C} \sum_{i, j \in I} \lambda_{i} \lambda_{j} f\left(y_{i}, y_{j}\right) \\
& =\frac{1}{2} \sum_{i, j \in I} \lambda_{i} \lambda_{j}\left\{f\left(y_{i}, y_{j}\right)+f\left(y_{j}, y_{i}\right)\right\} \\
& \leq_{C} 0 .
\end{aligned}
$$

On the other hand, the convexity of $\tau$ in the second variable and $C$-monotone imply that

$$
\begin{aligned}
\sum_{i \in I} \lambda_{i} \tau\left(y_{i}, u\right) & \leq_{C} \sum_{i, j \in I} \lambda_{i} \lambda_{j} \tau\left(y_{i}, y_{j}\right) \\
& =\frac{1}{2} \sum_{i, j \in I} \lambda_{i} \lambda_{j}\left\{\tau\left(y_{i}, y_{j}\right)+\tau\left(y_{j}, y_{i}\right)\right\} \\
& \leq_{C} 0 .
\end{aligned}
$$

Furthermore,

$$
\begin{aligned}
0 & =\langle T(u), u-u\rangle=\left\langle T(u), \sum_{i \in I} \lambda_{i} y_{i}-\sum_{i \in I} \lambda_{i} u\right\rangle \\
& =\left\langle T(u), \sum_{i \in I} \lambda_{i}\left(y_{i}-u\right)\right\rangle \\
& =\sum_{i \in I} \lambda_{i}\left\langle T(u),\left(y_{i}-u\right)\right\rangle, \\
0 & =\langle u-u, D x-D z\rangle \\
& =\left\langle\sum_{i \in I} \lambda_{i} y_{i}-\sum_{i \in I} \lambda_{i} u, D x-D z\right\rangle \\
& =\left\langle\sum_{i \in I} \lambda_{i}\left(y_{i}-u\right), D x-D z\right\rangle \\
& =\sum_{i \in I} \lambda_{i}\left\langle\left(y_{i}-u\right), D x-D z\right\rangle .
\end{aligned}
$$

Combining (31), (32), and (33), we have

$$
\begin{aligned}
\sum_{i \in I} \lambda_{i} & \left\langle\left(y_{i}-u\right), D x-D z\right\rangle+\sum_{i \in I} \lambda_{i}\left\langle T(u),\left(y_{i}-u\right)\right\rangle \\
& -\sum_{i \in I} \lambda_{i} f\left(y_{i}, u\right)-\sum_{i \in I} \lambda_{i} \tau\left(y_{i}, u\right) \in C \Longrightarrow \sum_{i \in I} \lambda_{i} \\
& \cdot\left\{f\left(y_{i}, u\right)\right. \\
& +\tau\left(y_{i}, u\right)-\left\langle T(u),\left(y_{i}-u\right)\right\rangle \\
& \left.-\left\langle\left(y_{i}-u\right), D x-D z\right\rangle\right\} \in-C .
\end{aligned}
$$

From (31) and (34), we conclude that

$$
\begin{aligned}
\sum_{i \in I} \lambda_{i}\left\{f\left(y_{i}, u\right)+\tau\left(y_{i}, u\right)-\left\langle T(u),\left(y_{i}-u\right)\right\rangle\right. \\
\left.\quad-\left\langle\left(y_{i}-u\right), D x-D z\right\rangle\right\} \in \operatorname{int} C \cap(-C)=\emptyset,
\end{aligned}
$$


which is a contradiction. This follows that $u \in \bigcup_{i \in I} F\left(y_{i}\right)$ and hence $\operatorname{Co}\left\{y_{i}: i \in I\right\} \subseteq \bigcup_{i \in I} F\left(y_{i}\right)$. Thus, $F$ is a KKM mapping. From assumption $(v i)$, we can see that the family $\left\{\left(C_{i}, Z_{i}\right)\right\}_{i \in I}$ satisfies the condition which is, for all $i \in I$, there exists $k \in I$ such that

$$
\bigcap_{y \in C_{k}} F(y) \subset Z_{i}
$$

and therefore it is a coercing family for $F$. We deduce that $F$ satisfies all the hypothesis of Theorem 9. Therefore, we have

$$
\bigcap_{y \in C_{k}} F(y) \neq \emptyset .
$$

Hence, there exists $\tilde{x} \in K$ such that for any $y \in K$

$$
\begin{gathered}
f(y, \tilde{x})-\langle T(\tilde{x}), y-\tilde{x}\rangle+\tau(y, \tilde{x}) \\
-\langle y-\tilde{x}, D \tilde{x}-D z\rangle \notin \operatorname{int} C .
\end{gathered}
$$

Now applying Proposition 11, we obtain that there exists $\tilde{x} \epsilon$ $K$ such that for all $y \in K$

$$
\begin{array}{r}
f(\tilde{x}, y)+\langle T(\tilde{x}), y-\tilde{x}\rangle+\tau(\tilde{x}, y) \\
+\langle y-\tilde{x}, D \tilde{x}-D z\rangle \notin-\operatorname{int} C .
\end{array}
$$

Hence problem (5) admits a solution. This completes the proof.

Corollary 12. Let $K, C,\left\{\left(C_{i}, Z_{i}\right)\right\}_{i \in I}, f, \tau, T$, and $D$ satisfy all the assumptions of Theorem 10. In addition, if $C$ satisfies Condition $(C)$, then problem (6) is solvable; that is, there exists $\tilde{x} \in K$ such that for any $y \in K$

$$
\begin{aligned}
& f(\tilde{x}, y)+\langle T(\tilde{x}), y-\tilde{x}\rangle+\tau(\tilde{x}, y) \\
& \quad+\langle y-\tilde{x}, D \tilde{x}-D z\rangle \notin-(C \backslash\{0\}) .
\end{aligned}
$$

Proof. Suppose that $C$ satisfies Condition $(C)$. Then there is a pointed convex and closed cone $\widetilde{C}$ in $Y$ such that $C \backslash\{0\} \subseteq$ int $\widetilde{C}$. Therefore, it is not hard to see that $K, C,\left\{\left(C_{i}, Z_{i}\right)\right\}_{i \in I}$, $f, \tau, T$, and $D$ satisfy all the assumptions of Theorem 10 . It follows from Theorem 10 that

$$
\begin{aligned}
& f(\tilde{x}, y)+\langle T(\tilde{x}), y-\tilde{x}\rangle+\tau(\tilde{x}, y) \\
& \quad+\langle y-\tilde{x}, D \tilde{x}-D z\rangle \notin-\operatorname{int} \widetilde{C} ; \quad \forall y \in K .
\end{aligned}
$$

Since $-(C \backslash\{0\}) \subseteq-\operatorname{int} \widetilde{C}$, (41) yields the fact that there exists $\tilde{x} \in K$ such that

$$
\begin{aligned}
& f(\tilde{x}, y)+\langle T(\tilde{x}), y-\tilde{x}\rangle+\tau(\tilde{x}, y) \\
& \quad+\langle y-\tilde{x}, D \tilde{x}-D z\rangle \notin-(C \backslash\{0\}) ; \quad \forall y \in K .
\end{aligned}
$$

Therefore, problem (6) admits a solution. This completes the proof.

In the case of $\tau \equiv 0$ and $D \equiv 0$, we obtain the following corollaries.
Corollary 13 (see [5]). Let $K$ be a nonempty closed convex subset of a Hausdorff topological vector space X, Y a Hausdorff topological vector space, and $C$ a closed convex pointed cone in $Y$ with int $\neq \neq \emptyset$. Let $f: K \times K \rightarrow Y$ and $T: K \rightarrow L(X, Y)$ be two mappings satisfying the following conditions:

(i) $f$ is C-monotone.

(ii) $f(x, x)=0, x \in K$.

(iii) For any fixed $x, y \in K, t \in[0,1] \mapsto f(t y+(1-t) x, y) \in$ $Y$ is upper semicontinuous with respect to $C$ at $t=0$.

(iv) For any fixed $x \in K, f(x, \cdot): K \rightarrow Y$ are $C$-convex, lower semicontinuous with respect to $C$ on $K$.

(v) $T$ is upper semicontinuous with respect to $C$ with nonempty closed values.

(vi) There exists a family $\left\{C_{i}, Z_{i}\right\}_{i \in I}$ satisfying conditions ( $i$ ) and (ii) of Definition 7 and the following condition: For each $i \in I$, there exists $k \in I$ such that

$$
\begin{aligned}
& \left\{x \in K: f(y, x)-\langle T x, y-x\rangle \notin \operatorname{int} C, \forall y \in C_{k}\right\} \\
& \quad \subset Z_{i} .
\end{aligned}
$$

Then, there exists a point $\tilde{x} \in K$ such that

$$
f(\tilde{x}, y)+\langle T(\tilde{x}), y-\tilde{x}\rangle \notin-\operatorname{int} C ; \quad \forall y \in K .
$$

Corollary 14 (see [5]). Let $K, C,\left\{\left(C_{i}, Z_{i}\right)\right\}_{i \in I}, f$, and $T$ satisfy all the assumptions of Corollary 13. In addition, if $C$ satisfies Condition $(C)$, then problem (4) is solvable; that is, there exists $\tilde{x} \in K$ such that for any $y \in K$

$$
f(\tilde{x}, y)+\langle T(\tilde{x}), y-\tilde{x}\rangle \notin-(C \backslash\{0\}) .
$$

\section{Conflict of Interests}

The authors declare that there is no conflict of interests regarding the publication of this paper.

\section{Acknowledgments}

The authors would like to thank Professor Dr. Tomonari Suzuki and two anonymous reviewers for their valuable comments and suggestions, which were helpful in improving the paper. The second author would like to thank Naresuan University. This research is supported by Naresuan University and the Centre of Excellence in Mathematics, the Commission on Higher Education, Thailand.

\section{References}

[1] E. Blum and W. Oettli, "From optimization and variational inequalities to equilibrium problems," The Mathematics Student, vol. 63, no. 1-4, pp. 123-145, 1994.

[2] X. P. Ding, "Quasi-equilibrium problems in noncompact generalized convex spaces," Applied Mathematics and Mechanics, vol. 21, no. 6, pp. 637-644, 2000.

[3] F. Giannessi, Vector Variational Inequalities and Vector Equilibria, Kluwer Academic Publishers, Dordrecht, The Netherlands, 2000 . 
[4] A. Moudafi, "Mixed equilibrium problems: sensitivity analysis and algorithmic aspect," Computers \& Mathematics with Applications, vol. 44, no. 8-9, pp. 1099-1108, 2002.

[5] M. Rahaman and R. Ahmad, "Weak and strong mixed vector equilibrium problems on non-compact domain," Journal of the Egyptian Mathematical Society, vol. 23, no. 2, pp. 352-355, 2015.

[6] G. M. Lee, D. S. Kim, and B. S. Lee, "On non-cooperative vector equilibrium," Indian Journal of Pure and Applied Mathematics, vol. 27, no. 8, pp. 735-739, 1996.

[7] M. Bianchi, N. Hadjisavvas, and S. Schaible, "Vector equilibrium problems with generalized monotone bifunctions," Journal of Optimization Theory and Applications, vol. 92, no. 3, pp. 527-542, 1997.

[8] F. Flores-Bazán and F. Flores-Bazán, "Vector equilibrium problems under asymptotic analysis," Journal of Global Optimization. An International Journal Dealing with Theoretical and Computational Aspects of Seeking Global Optima and Their Applications in Science, Management and Engineering, vol. 26, no. 2, pp. 141166, 2003.

[9] J. Fu, "Simultaneous vector variational inequalities and vector implicit complementarity problem," Journal of Optimization Theory and Applications, vol. 93, no. 1, pp. 141-151, 1997.

[10] N. X. Xuan and P. N. Nhat, "On the existence of equilibrium points of vector functions," Numerical Functional Analysis and Optimization, vol. 19, no. 1-2, pp. 141-156, 1998.

[11] K. Fan, "A minimax inequality and applications," in Inequalities, O. Shisha, Ed., vol. 3, pp. 103-113, Academic Press, New York, NY, USA, 1972.

[12] Z. Al-Rumaih, S. Chebbi, and H. K. Xu, "Noncompact equilibrium points and applications," Journal of Applied Mathematics, vol. 2012, Article ID 373462, 9 pages, 2012.

[13] W. Oettli and S. Schläger, "Generalized vectorial equilibria and generalized monotonicity," in Functional Analysis with Current Applications, M. Brokate and A. H. Siddiqi, Eds., pp. 145-154, Longman, London, UK, 1997.

[14] G. Y. Chen, "Existence of solutions for a vector variational inequality: an extension of the Hartmann-Stampacchia theorem," Journal of Optimization Theory and Applications, vol. 74, no. 3, pp. 445-456, 1992.

[15] H. Ben-El-Mechaiekh, S. Chebbi, and M. Florenzano, "A generalized KKMF principle," Journal of Mathematical Analysis and Applications, vol. 309, no. 2, pp. 583-590, 2005. 


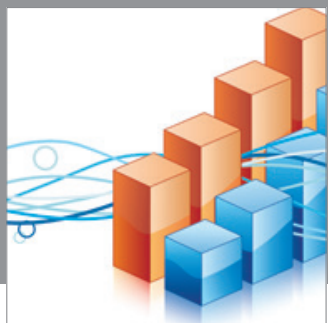

Advances in

Operations Research

mansans

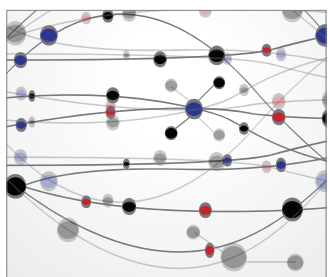

The Scientific World Journal
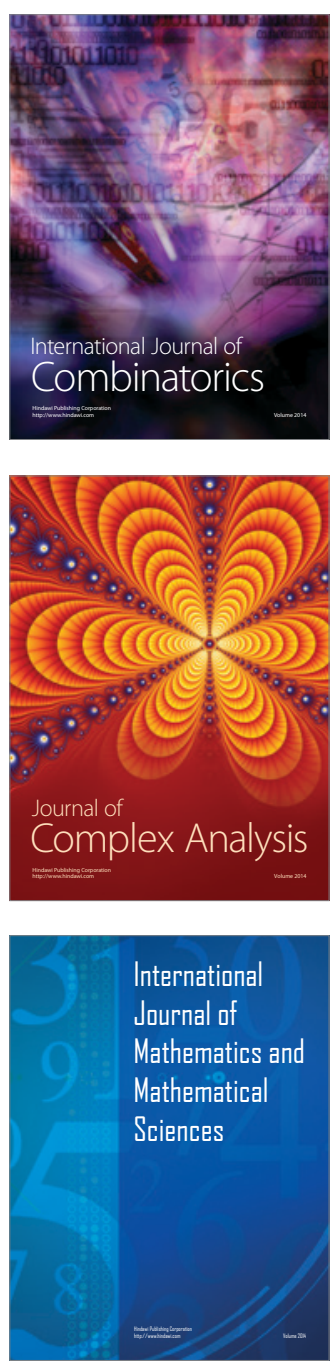
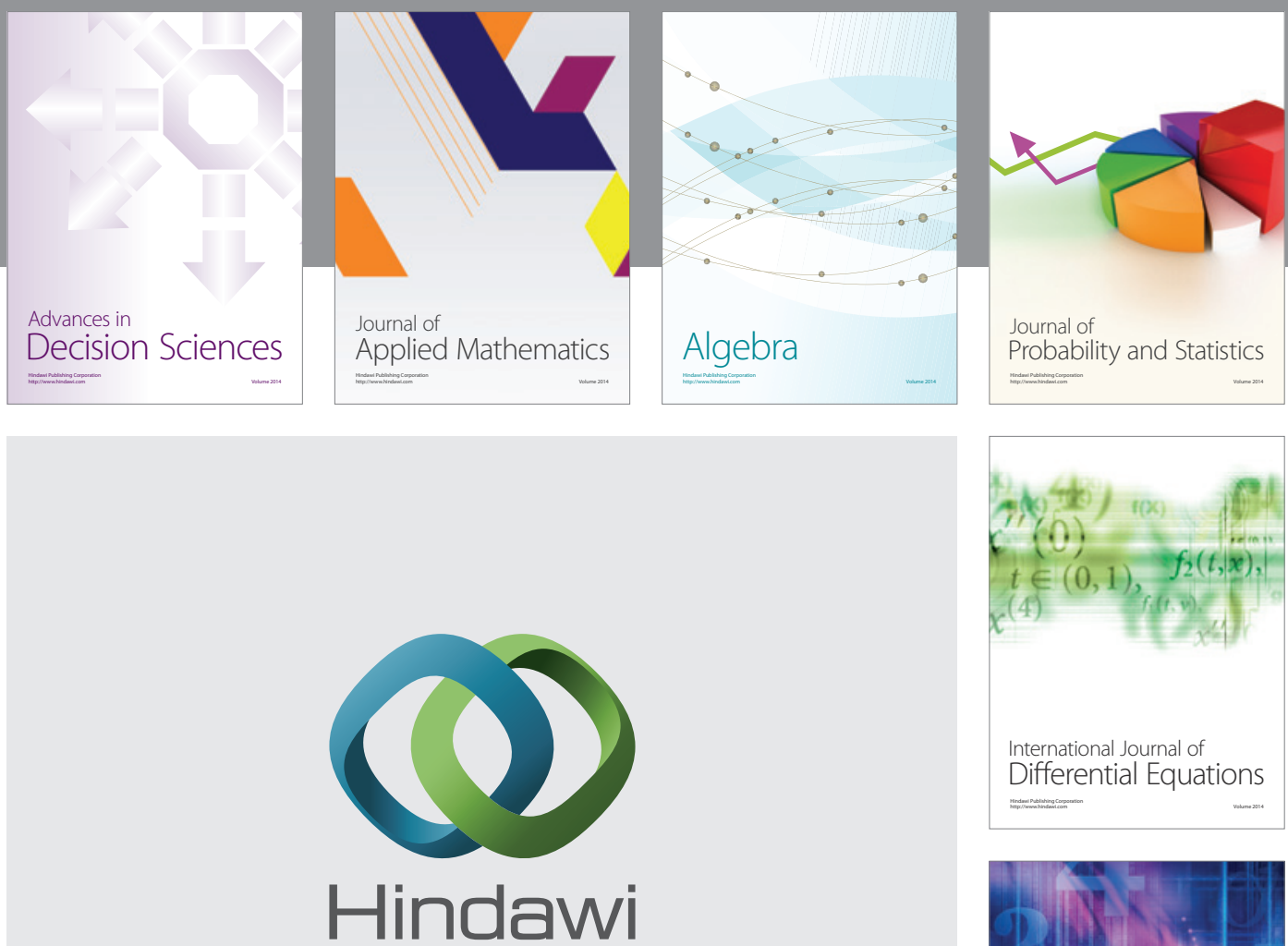

Submit your manuscripts at http://www.hindawi.com
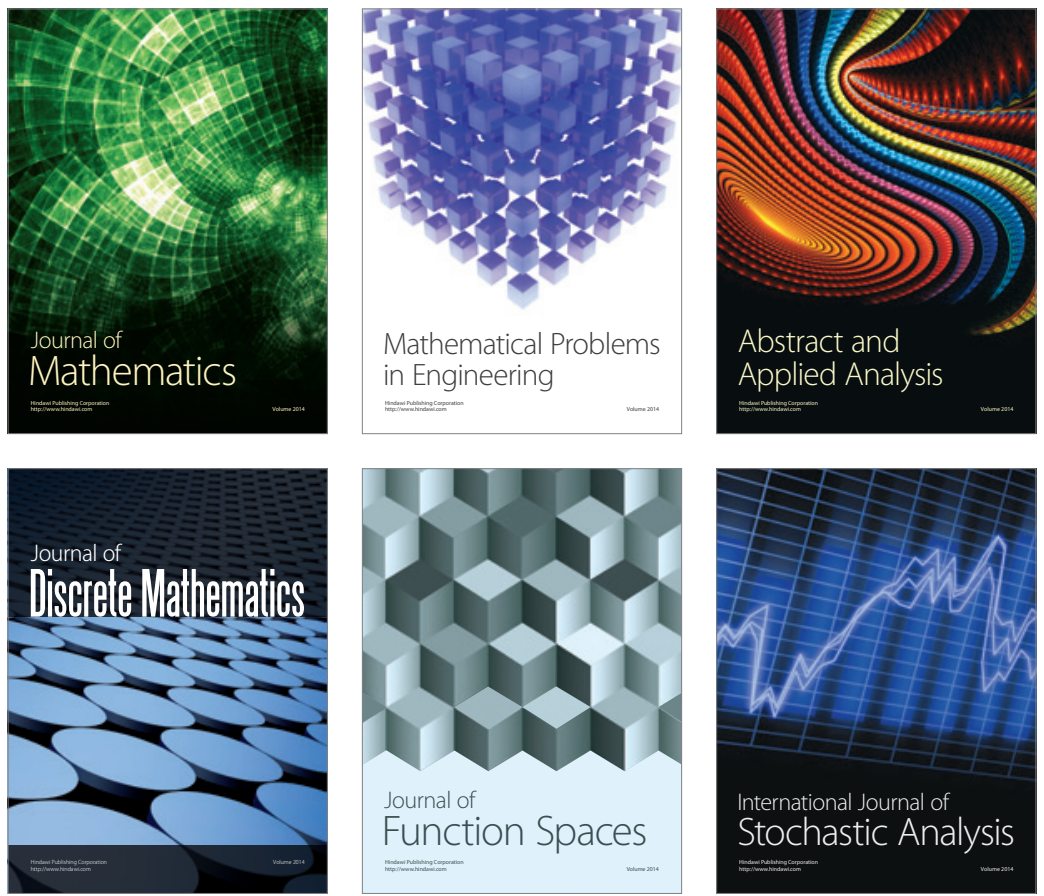

Journal of

Function Spaces

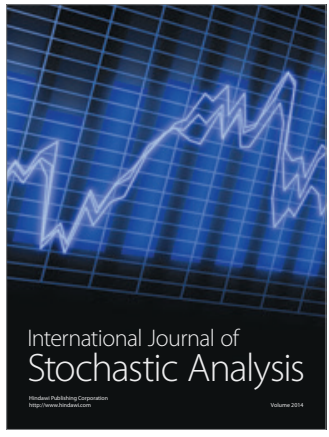

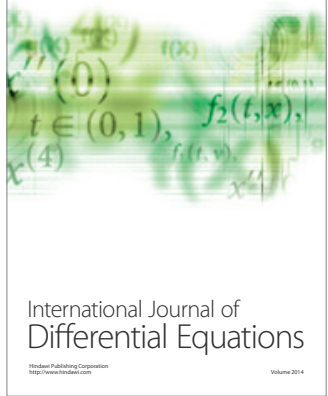
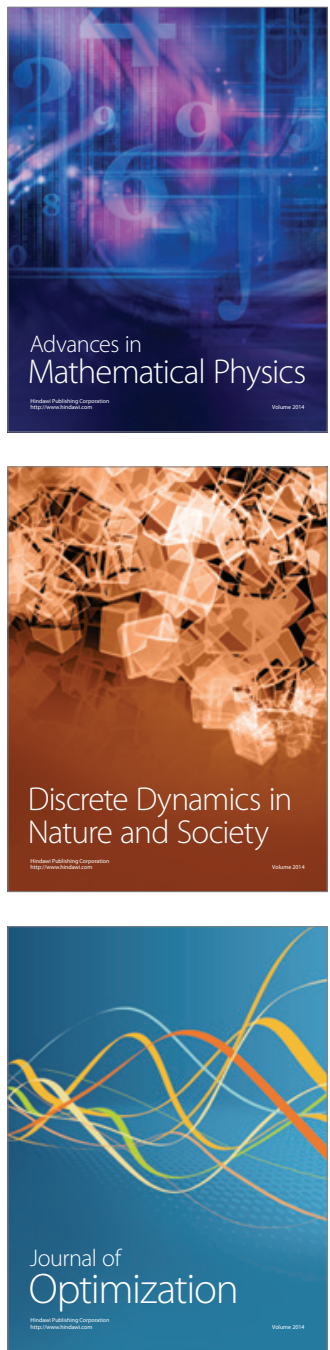